\title{
Estratégias construídas por mulheres negras para o enfrentamento das discriminações por raça e gênero no Brasil e no Canadá: desafios de um projeto de pesquisa
}

\begin{abstract}
Strategies built by black women for facing discriminations based
on race and gender in Brazil and Canada: challenges of a research

project
\end{abstract}

\author{
Gislene Aparecida dos Santos ${ }^{1}$ \\ Camila Tavares de Moura Brasil Matos ${ }^{2}$
}

Submetido em 5 de novembro e aprovado em 6 dezembro de 2018.

\begin{abstract}
Resumo: Neste artigo, apresentamos os desafios que enfrentamos para implementar o projeto de pesquisa denominado "Estratégias construídas por mulheres negras para o enfrentamento das discriminações por raça e gênero no Brasil e no Canadá: desafios de um projeto de pesquisa", encaminhado à SECADI / CAPES nº. 02/2014 - Programa de desenvolvimento acadêmico Abdias Nascimento. Em perspectiva comparada, o objetivo deste projeto foi investigar se as mulheres negras construíram ou desejariam construir estratégias de enfrentamento de experiências de discriminação racial; e descrever quais seriam essas estratégias. No entanto, em vez de apresentar os resultados da investigação realizada até agora, decidimos discutir o projeto de pesquisa em si. Acreditamos que a discussão de projetos de pesquisa pode ser uma fonte de novas propostas ajudando a compreender os desafios na condução de investigações e, também, fornecer informações sobre como superá-los. Com isso, organizamos este artigo em duas partes principais, com subpartes. Na primeira, apresentamos o projeto de pesquisa de acordo com os seguintes tópicos: a proposta, base teórica: estudos pós-coloniais e feminismo negro, questões norteadoras, objetivos, materiais e métodos. Na segunda parte, apresentamos e discutimos os desafios para definir quem seriam as mulheres negras, no Canadá, e sobre o desenvolvimento de subprojetos que permitiram resolver questões teóricas e práticas surgidas no dia a dia da investigação. Concluímos que é importante ter em mente as diferentes dimensões de opressão e dominação para obter sucesso em projetos de pesquisa bilaterais, focados em questões de mulheres negras.
\end{abstract}

Palavras-chaves: Mulheres negras. Feminismo negro. Interseccionalidade. Minorias visíveis. Projeto de pesquisa. Brasil-Canadá.

Abstract: In this article, we present challenges we faced to implement the research project named "Strategies built by black women for facing discriminations based on race and gender in Brazil and Canada: challenges of a research project", submitted to SECADI/CAPES no. 02/2014 - Abdias Nascimento academic development program. In comparative perspective, the goal of this project was to investigate whether black women built or wish to build coping strategies to face experiences of racial discrimination; and 
to describe what these strategies would be. However, instead of presenting findings of investigation carried out so far, we decided to discuss the research project itself. We believe that discussing research projects should be sources for new proposals, helping to comprehend challenges of conducting investigations and also, to produce information about how to overcome them. With that, we organized this article in two main parts, with subparts. In the first, we present the research project according to the following topics: proposal, theoretical basis: post-colonial studies and black feminism, guiding questions, objectives, materials and methods. In the second part, we present and discuss challenges regarding to define who were the black women in Canada, and about development of subprojects that allows to solve theoretical and practical questions emerged during the day by day of this investigation. We concluded that is important to keep in mind the different dimensions of oppression and domination in order to succeed in bilateral research projects focused in black women issues.

Keywords: Black women. Black feminism. Intersectionality. Visible minorities. Research Project. Brazil-Canada.

\section{Apresentação}

Embora abunde literatura que ensine como construir projetos de pesquisa, não há muitas publicações, sob a forma de artigos, que discutam os empasses no momento da realização da pesquisa. Por isso, escolhemos trazer esse tema para discussão. Acreditamos que a reflexão sobre isso pode ser fonte para o desenvolvimento de novas propostas e auxiliar na compreensão dos desafios que a investigação acadêmica envolve, com indicações sobre como superá-los.

Organizamos este artigo em duas partes principais, com subpartes. Na primeira, apresentamos o projeto de pesquisa proposto em acordo com os tópicos proposta, fundamentação teórica, questões norteadoras, objetivos, métodos e técnicas da pesquisa. Na segunda parte, apresentamos e discutimos os desafios encontrados para a implementação e realização da pesquisa e mostramos como foram superados.

No primeiro ano de realização da investigação, os desafios se concentraram em torno de dois fatores principais: o primeiro se referiu ao fato de que não havíamos considerado a diversidade envolvida no modo de conceituar "mulheres negras". Partimos do pressuposto de que, tanto no Brasil quanto no Canadá, o conceito "mulher negra" designaria as mesmas pessoas, sem distinção. Aprendemos que isso não é verdade. 
O segundo fator se vinculou ao modo como o projeto foi estruturado. Os objetivos foram definidos para serem cumpridos por uma única equipe de trabalho concentrada na realização das várias etapas da pesquisa. Contudo, ao nos depararmos com as questões teóricas e conceituais que surgiram, percebemos que esses objetivos, na verdade, eram muito mais amplos e exigiriam equipes diferentes que se concentrassem em aspectos pontuais da pesquisa. Por isso, foi necessário desdobrarmos o projeto principal em subprojetos por meio dos quais fossem investigados esses aspectos que contribuiriam, ao final, com argumentos auxiliares na resposta das questões do projeto principal. Apresentaremos os subprojetos mais a frente.

\section{I - O projeto de pesquisa}

\section{1- A proposta}

Fruto de uma parceria de pesquisa entre a Universidade de São Paulo - Diversitas - Núcleo de Estudos das Diversitas, Intolerâncias e Conflitos, Brasil e a University of Toronto-Centre for Integrative Studies Anti-Racism, CIARS-OISE/UofT, Canadá, o objetivo deste projeto, era e é o de documentar o impacto do racismo nas mulheres negras e modos de enfrentamento. Por meio deste projeto, se desejou instituir a parceria entre o Diversitas e o CIARS, associada ao Memorandum of Understanding assinado entre a University of Toronto e a Universidade de São Paulo, que comprometeu as duas universidades a colaborarem em projetos de pesquisa, intercâmbios de estudantes, bem como a co-realização de palestras, reuniões, seminários, simpósios e conferências.

A pesquisa- joint-venture USP-UofT ainda está em execução. Mas, neste texto, trataremos do que já foi realizada pelas estudantes selecionadas, supervisionadas pelas coordenadoras do projeto, e que teve como foco investigar e analisar estratégias de enfrentamento de discriminações vivenciadas por mulheres brasileiras (no Brasil) e por mulheres canadenses (no Canadá).

Por se tratar de uma parceria bilaterial, dividimos o projeto em duas fases complementares. A primeira, coordenada pela Professora Gislene Aparecida dos Santos, a ser realizada no Brasil, implicava analisar estratégias de enfrentamento construídas por mulheres negras que foram alvos de injúria racial ou racismo (situações de violência 
e humilhação), cujos nomes constavam do banco de dados formado por nós durante a pesquisa realizada entre 2012-2014, com financiamento da FAPESP³.

Do lado canadense, a pesquisa coordenada por Njoki Wane teria como foco analisar a contribuição que as teorias feministas têm oferecido para a contrução de estratégias de enfrentamento da discriminação de raça e gênero de que são alvos as mulheres negras canadenses e de ascendência africana que vivem no país. Também objetivava identificar que tipo de treinamento/formação pode ser oferecido para educadores, estudantes, agentes comunitários, assistentes sociais no sentido de fazer uso de estratégias de enfrentamento adequadas para auxiliarem as mulheres negras a lidarem com as situações de discriminação em razão de sua cor, raça e gênero e, ainda conhecer experiências de sucesso descritas por essas mulheres.

Definimos como "estratégias de enfrentamento" os modos criados ou buscados por essas mulheres para lidarem com situações de discriminação. Queríamos e queremos saber se essas mulheres utilizaram ou desejariam utilizar, construíram ou desejariam construir essas estratégias de enfrentamento para auxiliá-las a lidar com esta situação de humilhação e violência, se desejavam auxílio para criá-las, de que forma e quais estratégias considerariam adequadas. Além disso, nos interessava obter informações que permitissem entender se as soluções de enfrentamento encontradas pelas mulheres negras do Canadá seriam similares às indicadas pelas mulheres do Brasil. Se similares, quais as razões que explicariam essa semelhança? Se diversas, uma experiência poderia contribuir para o aprimoramento da outra?

Vinculamos esse projeto à linha temática do programa Abdias do Nascimento voltada à "Educação e Desigualdade/Desigualdade étnico-raciais no Brasil contemporâneo/Estudos sobre interculturalidade na universidade/Relações étnico -raciais", recortando a discussão para uma perspectiva de gênero. E orientamos nossa reflexão pelo campo teórico/epistemológico dos estudos pós-coloniais.

\section{2- Fundamentação teórica: estudos pós-colonais e feminismo negro}

Os estudos pós-coloniais têm raízes antigas. Na década de 1950, Frantz Fanon em seu livro Pele Negra, Máscaras Brancas (um dos marcos dos estudos pós-coloniais) já 
sinalizava para as tensões psíquicas e políticas que se entrelaçavam na construção dialética das identidades negras e brancas a partir de discursos que negavam o reconhecimento do negro. (FANON, 1983).

No Canadá, Njoki Wane, tem se dedicado ativamente ao estudo do antirracismo e da construção de estratégias para que mulheres negras lidem com situações de discriminação. A pesquisadora tem publicado sobre o papel das mulheres na produção do conhecimento com o objetivo de revelar a participação, até então não reconhecida, de mulheres negras na manutenção de suas comunidades. Com esse propósito, através de financiamento do SSHRC (Social Sciences and Humanities Research Council), entrevistou mulheres negras no Canadá, assim como mulheres africanas no Quênia para compor sua teoria sobre feminismo negro. Esta teoria é discutida nos cursos que ministra na University of Toronto e nos grupos de suporte a mulheres negras, em Toronto.

A autora conceitua o feminismo negro como um quadro teórico que ilustra as experiências históricas, sociais, políticas, culturais e econômicas das mulheres negras, no Canadá (WANE, 2002, 2004, 2007) mas que assume uma perspectiva diaspórica, internacional, já que se baseia no estudo das narrativas de mulheres negras imigrantes, residentes, de diferentes partes da África, mas também se apoia no estudo das teorias desenvolvidas por diferentes autoras que se debruçaram sobre o tema gênero e raça em diferentes países de língua inglesa. Como a autora não estudou autoras feministas brasileiras, este projeto também pode ser uma oportunidade para que os pesquisadores canadenses descubrissem, conhecessem e incorporassem autores e autoras brasileiras a sua literatura sobre o feminismo negro.

A partir destas experiências focalizadas na sabedoria, habilidades e esforços de mulheres negras, Wane definiu os princípios fundamentais do feminismo negro desenvolvido no Canadá como sendo: a organização, o coletivismo, a resistência, o respeito mútuo, a produção de conhecimento, o armazenamento do conhecimento, divulgação da cultura, a reciprocidade, a auto-determinação, resiliência, cuidados com a comunidade, maternagem, fortalecimento mútuo, auto-confiança e espiritualidade.

Acredita que o termo "mulheres negras" captura uma heterogeneidade e diferenças complexas e dizem respeito a todas as mulheres negras que vivem em 
uma sociedade racializada e multicultural como é a canadense, mas como também é a brasileira e muitas outras. Nessas sociedades, há uma intersecção de opressões em um espaço predominantemente branco, eurocentrado, onde as mulheres negras são praticamente invisíveis.

No Canadá, há uma enorme produção sobre teoria feminista que discutem estratégias de resistência. Segundo Wane, nos últimos cinco anos, muitos pesquisadores têm teorizado sobre esse tema: Lawson 2002; Tastsoglou, 2002; Deliovsky 2002; Elabor-Idemudia, 2001; Wane, 2002, 2004, 2007; Riviere, 2004; Massaquoi, 2004, 2007; Crawford, 2004. Esses trabalhos somam-se a outros que focalizam temas como sexualidade, racismo, maternagem, liderança comunitária, ativismo, produção acadêmica (CARTY, 1991; HENRY, 1998), mercado de trabalho (BRAND, 1991; CARTY, BRAND, 1993), violência (MAKEDA, 1989), saúde das mulheres e história (SHADD, 1994), cultura (ELABOR-IDEMUDIA, 2001), polícias imigrantistas (DEI, CALLISTE, 2000), e experiencias de mulhetes negras de ascendência africana no Canadá.

Essas obras ilustram as descobertas que as pesquisadoras negras canadenses fizeram no sentido de compreender os desafios necessários para o enfrentamento do racismo, do classismo dentro dos movimentos feministas tradicionais, e compreender o sexismo e a homofobia dentro das comunidades negras canadenses. Também permitem compreender como o nacionalismo, o fundamentalismo religioso e a política de imigração seguem parâmetros discriminatórios; compreender os privilégios associados à brancura e a exploração econômica que são transversais a todos esses fenômenos (BRAND, 1991; CARTY, 1991; HENRY, 1998).

Fizemos uso destas teorias do feminismo negro aqui apresentadas para a construção de categorias que explicitassem estratégias de enfrentamento das experiências de discriminação racial de que foram alvos mulheres negras nos dois países.

Mesmo estando em locais diferentes, com histórias diversas, pensamos que as experiências de opressão, violência e discriminação racial unificassem as mulheres negras dos mais diversos locais como se houvesse uma comunidade de destino ou como se formassem o mesmo grupo social já que partilhariam histórias semelhantes de opressão. 
Não podemos esquecer de que o ponto de partida desta investigação, no Brasil, foram os depoimentos de mulheres negras cujos nomes constam como vítimas ${ }^{4}$ e autoras de queixas-crimes em processos e inquéritos com conteúdo raciais ocorridos em São Paulo - Capital, entre 2003 e 2011 que fazem parte do banco de dados criado pelo GEPPIS 5 . Esse banco de dados é composto por 119 documentos aos quais tivemos acesso, ao longo do ano de 2012 e $2013^{6}$. Trata-se aqui de analisar fatos julgados de opressão e de refletir sobre se o mesmo ocorreria no Canadá.

Pudemos observar que grande parte das ofensas que resultaram em procedimentos judiciários foram praticadas contra mulheres e envolviam a dupla discriminação por raça e gênero. (Neste banco de dados, 53\% representavam denúncias feitas por homens e $47 \%$ denúncias feitas por mulheres). Na maioria das vezes, as ofensas raciais contra as mulheres negras recobriam caracteres de animalidade e vulgaridade sexual (preta, vagabunda, macaca, puta, negrinha burra, é porque você é negra, urubu, negra fedida, besouro, galinha, negra suja).

Diante das ofensas sofridas, de modo geral, homens e mulheres apresentaram os sentidos de indignação, mágoa, rancor, raiva, ódio, inconformidade, chateação, frustação impotência, vergonha, humilhação, nervosismo, constrangimento, desorientação.

Emoções semelhantes a essas foram manifestadas na pesquisa conduzida por Oliveira, Meneguel e Bernandes (2009) que investigaram as estratégias de enfrentamento construídas por mulheres que fizeram denúncias de racismo no SOS racismo da cidade de Porto Alegre. As autoras relatam que as participantes de seu estudo fizeram menção a um amplo repertório: o da denúncia do racismo e da discriminação racial, o dos efeitos do racismo em relação às identidades e subjetividades e, mencionaram também as estratégias que utilizam para enfrentar a discriminação racial. Assim, “(...) as mulheres narraram situações de violência racial que motivaram a busca de ajuda em depoimentos carregados de emoção, com falas que diziam da tristeza, vergonha, culpa e do medo da repetição da agressão" (OLIVEIRA, MENEGUEL, BERNANDES, 2009, p. 269).

Seria possível afirmar que o mesmo ocorreria com mulheres que vivem no hemisfério norte em um país reconhecido por sua diversidade cultural, por adotar e assumir o multiculturalismo? 
Essas descobertas nos conduziram a novas perguntas sobre a situação destas mulheres: se teriam desenvolvido estratégias específicas para lidarem com essa humilhação, se acreditavam que seria necessário criar estas estratégias. Também observamos que havia pouca produção acadêmica focalizada nos componentes psicológicos ligados à vulnerabilidade das mulheres negras em sociedades racistas de modo a sugerir estratégias de enfretamento do estresse gerado pelo racismo. Tampouco havia pesquisas que relacionassem racismo e estresse, sendo que esses aspectos somente muito recentemente passaram a ser considerados como importantes em termos da saúde coletiva.

Contudo, autores como Sampaio (2009); Nascimento, Sardinha e Pereira (2012), Machado, Pires e Lobão (2012), Fonseca et. al. (2009) estudaram e avaliaram a relação da hipertensão em mulheres negras brasileiras indicando que: 1- há grande incidência de hipertensão arterial em mulheres negras sendo essas as maiores vítimas deste tipo de enfermidade; 2- há relação entre estresse, ansiedade e fatores emocionais com o aumento de doenças hipertensas e cardiovasculares.

Fora do Brasil, estudos apontam que esse tipo de opressão, ligado à discriminação racial, tem sido relatado como causa de grande estresse. West, Donovan, Roemer (2010) discutem como as mulheres negras lidam (ou não) com o estresse relacionado à raça e o que isso significa, em termos de sintomas de saúde física e saúde mental.

Para elas, existe uma grande quantidade de microagressões contra populações negras. Perceber a discriminação racial implica um alto custo psicológico e físico associados à hostilidade, doenças somáticas, ansiedade e depressão. Viver esta discriminação e realizar o esforço de denunciá-la pode aumentar ainda mais o estresse.

Do mesmo modo, autoras relacionam a construção de estratégias para lidar com estresse com estratégias de coping 7 . West, Donovan, Roemer (2010) mencionam que a relação entre gênero e coping, dentro da comunidade negra, ainda é pouco explorada. De acordo com elas: "coping style refers to the specific efforts, both behavioral and psychological, that people use to minimize stressfull events"8 (WEST, DONOVAN e ROEMER, 2010, p. 333). Em seus estudos, sugerem que o coping tende a minimizar os sintomas de depressão entre mulheres negras, e se recusar ao coping implica altos níveis de depressão. 
Além desta perspectiva associando gênero, raça e coping, encontramos autoras que, como Greer (2011), mostram que ainda deve ser explorada a maneira como as mulheres negras lidam com o estresse da discriminação de raça e gênero.

Em sociedade coloniais, patriarcais e racistas, a discriminação e a humilhação precisam ser desnudadas por novas produções de conhecimento como demonstrado por Santos (2002a, 2002b, 2007, 2008, 2012) e por Oliveira, Meneguel e Bernandes (2009, p. 272):

Acreditamos que essa pesquisa trouxe algumas contribuições para compreender os efeitos da discriminação racial nas identidades e subjetividades das mulheres negras. A construção do conhecimento sobre a violência racial, produzida à luz dos pressupostos teóricos da psicologia social e da saúde coletiva, precisa ser incorporada à agenda das políticas públicas para o combate à violência contra as mulheres, considerando as especificidades das mulheres negras. Essa foi uma pesquisa com aspectos de intervenção psicossocial, pois não esquecemos que a pesquisa é dotada de caráter político, e que a ciência é uma prática social.

Do mesmo modo, as histórias dos povos negros e, neste caso especificamente das mulheres negras, precisam ser contadas, descobertas e compreendidas.

\section{3 - Questões norteadoras, objetivos, materiais e métodos}

Considerando todos esses aspectos, as questões que proposemos para nortear essa investigação foram: se as mulheres negras construíram ou desejam construir estratégias de enfrentamento de experiências de discriminação racial? Quais seriam essas estratégias?

Consideramos que responder a essas perguntas fosse essencial em razão da baixa produção acadêmica em torno da subjetividade das mulheres negras. Também porque desejávamos saber mais sobre as especificidades das discriminações que podem atingilas e conhecer as estratégias que pudessem adotar para enfrentar situações de estresse, violência e humilhação decorrentes de discriminações que interseccionassem elementos de raça/cor/gênero. A resposta a essas questões permitiria relacionar estresse e racismo, as dores relativas a isso e a estratégias pessoais e sociais construídas. Deste modo, a investigação contribuiria com informações importantes para o desenvolvimento de políticas de atenção à saúde psicossocial das mulheres negras, políticas educacionais, 
de assistência comunitária e social colaborando para o fortelecimento dos vínculos entre famílias, comunidades, escolas, tudo o que, acreditamos poderíamos aprender ao longo e por meio da pesquisas realizadas no CIARS, University of Toronto.

No projeto, definimos como objetivo geral conhecer e descrever estratégias de enfrentamento da discriminação racial construídas por mulheres negras. Consideramos como objetivos específicos: 1- saber se as mulheres negras possuiriam estratégias de enfrentamento de experiências de discriminação racial; 2 - documentar e descrever quais estratégias indicariam para esse propósito; 3- verificar se haveria coincidência entre as estratégias mencionadas no Brasil e as desenvolvidas no Canadá; 4- promover o estudo e a divulgação das teorias pós-coloniais, do feminismo negro e dos estudos de gênero.

Para atender a esses objetivos, conjugamos técnicas que permitissem que o fenômeno investigado por nós fosse descrito, discutido e compreendido. Optamos pelo uso das seguintes técnicas de pesquisa: pesquisa bibliográfica, pesquisa documental no CIARS e a realização de entrevistas semiestruturadas, no Brasil.

Em um primeiro momento, definimos que o método de análise de conteúdo seria o ideal para tratar o material coletado o qual seria organizado em categorias definidas tanto a priori (antes da entrada no campo, já na formulação do projeto de pesquisa), quanto a posteriori, após a leitura flutuante de todo o material que comporia o corpus da pesquisa.

Com esse desenho começamos a realizar a pesquisa aqui apresentada.

\section{II- DESAFIOS ENCONTRADOS}

\section{1. - Quem são as “mulheres negras" canadenses?}

A pesquisa empírica se iniciou em 2017 com o envio de estudantes para realizar investigações, durante um ano, na University of Toronto, a fim de coletar dados sobre as mulheres negras canadenses e compreender melhor a existência do racismo e do multiculturalismo naquele país.

Como nós iremos demonstrar, em que pese os caminhos para desenvolvimento da pesquisa terem sido delineados por meio da criação de uma metodologia voltada à realização de entrevistas, análise de documentos e pesquisa bibliográfica, a realidade é que a coleta de dados em si se transformou em um desafio tendo em vista as diferenças 
no entendimento sobre como pesquisar sobre raça e gênero no Brasil, em oposição ao que ocorre no Canadá.

Na construção do feminismo canadense, o racismo não foi discutido extensivamente. Assim como ocorreu nos Estados Unidos, durante a primeira onda do feminismo, foi assumida a posição de que todas as mulheres compartilhavam as mesmas dificuldades e anseios. Porém, para as "mulheres de cor", a teoria feminista que estava em voga acabou por ignorar raça e classe, além de reproduzir as relações de dominação e subordinação presentes na sociedade (DAVIS, 2016).

Embora as opressões sofridas em função do racismo, classismo, sexismo e machismo sejam comuns e possam ser abordadas sob a ótica da interseccionalidade tanto no Brasil quanto no Canadá, a mobilização de mulheres negras em um país multicultural e desenvolvido como o Canadá, em contraste com a vivência e a mobilização de mulheres negras em países em desenvolvimento pertencentes à América Latina, como o Brasil, colocam desafios para a compreensão sobre a dinâmica na construção das identidades, culturas, raças e cores, em cada nação.

Sabemos que raça não é um marcador fixo da identidade. A definição sobre o pertencimento racial varia de acordo com os lugares em que se habita (MAIA, 2009). Deste modo, embora não tenhamos considerado as diferenças em relação as variações nas categorias gênero (mulher cis, trans, por exemplo), observamos as variações em torno da categoria "mulher negra".

Esse aspecto impactou inclusive no processo seletivo das pessoas que iriam participar do projeto já que o que entendíamos como preto, pardo, branco no Brasil (dados que constaram no documento de autodeclaração solicitado dos pesquisadores inscritos, por exigência do edital ao qual o projeto se vincula), assumia outro sentido no Canadá.

No caso dos negros no Canadá, esses estão incluídos dentro do conceito de visible minorities, ou seja, minorias visíveis, conforme estipulado no Employment Equity Act. Assim sendo, minorias visíveis é um termo de uso oficial para definir políticas, e são aquelas, além dos povos aborígenes, que não são caucasianos em raça ou não são brancos na cor ${ }^{9}$. Ou seja, sul-asiáticos, chineses, negros, filipinos, latino-americanos, árabes, pessoas do sudeste e do oeste asiático, coreanos e japoneses, ou qualquer outro 
grupo que se enquadre em minorias visíveis. Todas as pessoas dentro deste amplo grupo podem se referir a si mesmas como pertencentes a grupos vulneráveis, como pessoas portadoras de 'cor', racializadas e alvos de discriminação em razão de seu pertencimento étnico, por exemplo.

No Black Experience Project (BEP), elaborado pelo instituto Environics no Canadá, é dito que raça representa apenas um aspecto da identidade. Para nós, além disso, raça é uma construção social e política, resultante da estratificação das sociedades (SMEDLEY, 1993). Uma pessoa ser de origem africana, caribenha, ou canadense também é considerada uma forma de identidade ou etnia (identity and ethnicity). A cor é um dos elementos mais importantes. O resultado do BEP parece indicar o caminho de que o racismo acontece em função da cor e a afirmação de discriminação because we are black (porque você é negro) se mantém firme ao longo do relatório. Contudo, outros fatores também podem aparecer como fundamentais para a definição da identidade e surgirem no momento em que se pergunta sobre o pertencimento e identificação das pessoas que queremos que participem de nossas investigações.

O censo populacional do Canadá faz a contabilização da população por meio de questionários não em função da cor, mas em função de grupos (population group question) por meio de auto identificação ${ }^{10}$, sendo que a categoria black é uma delas. Outras categorias podem ser, south asian, chinese, filipino, latin american, arab, southeast asian, korean, japanese, west asian, além daquelas que não podem ser enquadrados nestes grupos como misturas étnicas como tibetanos, polinésios, guyaneses etc. Como dito, todos podem ser considerados minorias e vulneráveis, deste modo, a inserção dos negros dentro desta categoria genérica e ampla cria inúmeros obstáculo para a coleta de informações específicas sobre a população negra. Isso é bastante diferente do que ocorre no Brasil de hoje no que diz respeito a coleta de informações, via censo, pautados em cores com preto, pardo, branco, amarelo e indígena.

Talvez por isso seja bastante complicada a definição de quem são as mulheres negras, no Canadá. A definição do que é ser mulher negra canadense ainda está em construção, sobretudo por meio dos estudos esposados por Wane em Ruptures: anticolonial \& anti-racist feminist theorizing (2013). A autora salienta que as mulheres 
negras canadenses devem conceituar seu próprio feminismo, nos mostrando que devem colocar suas experiências e ideias no centro da análise do que é ser feminista, justamente porque, o feminismo ocidental não dialoga com as mulheres negras, assim como a teoria feminista negra norte-americana, muitas vezes, não se comunica com a canadense.

Observa-se, desta forma, que há definições oficiais (que são as bases das políticas públicas) e por meio das quais as pessoas podem indicar suas identidades por meio de vários atributos que vão da cor, ao pertencimento geográfico, cultural ou étnico (além das questões de gênero que não estamos especificando neste texto). Mas há, também, as definições construídas para a identidade das mulheres negras por meio de seu ativismo social. Ou seja, uma identidade que é, acima de tudo, política, edificada no seio das disputas feministas.

Wane expõe que seus alunos chegaram à conclusão de que o Black Canadian Feminist Thought (obra da autora em parceira com seus alunos) é uma "ferramenta teórica destinada a esclarecer a analisar os relacionamentos históricos, sociais, culturais e econômicos das mulheres afro-descendentes como base para desenvolvimento de uma práxis libertadora" $\left(2013\right.$, p. 38) ${ }^{11}$. Ser mulher negra, torna-se, desta forma, ser parte desta construção sócio-política que liberta, emancipa e transforma a realidade social.

Contudo, essa definição de quem seriam as mulheres negras por meio de uma identidade política não anula o fato de que, na prática, essas mulheres continuam a se referirem a si mesmas de múltiplas formas como black canadian, ou african canadian ou black caribbean. Ou ainda, que essas mulheres podem alterar seu modo de identificação em momentos diferentes de suas vidas, considerando sua mobilidade social, o status conquistado ou a manutenção de sua identificação com sua família de origem (KESLER \& SCHWARTZMAN, 2015). Resta a pergunta de quais mulheres negras estaríamos falando quando perguntamos sobre suas identidades para que possamos coletar, a partir daí, informações para nossas investigações.

Na pesquisa conduzida por Wane, a estratégia utilizada pela autora foi a de agrupar todas as diferentes identidades ${ }^{12}$ em uma só por ela denominada black canadian woman. Se essa estratégia facilita a categorização para efeitos de análise, cria, contudo, problemas para um estudo adequado das múltiplas facetas e dimensões que se fazem presentes na 
realidade social. Entretanto, na impossibilidade de listar, por meio de um só conceito ou categoria, todas essas experiências, Wane cunhou essa expressão com ressalva de que o objetivo não era achatar as experiencias vividas, mas incorporá-las.

O principal ponto de referência que se discute é justamente o significado de negritude em um país como o Canadá e como isso pode ser usado como um marcador da diferença para o combate ao racismo e ao sexismo. Como podemos traduzir esses aspectos em pesquisas acadêmicas? Se iríamos assumir a categorização definida por Wane de que todas as mulheres negras seriam, em resumo, mulheres negras canadenses; se iríamos assumir a definição de uma identidade construída na luta política por meio da qual as mulheres negras são aquelas que lutam pela liberdade, se iríamos adotar as múltiplas definições encontradas nas plataformas estatais?

Ao longo da pesquisa, várias teóricas do hemisfério norte que discutem o tema foram estudadas em busca de ideias que nos auxiliassem no processo de categorização e realização, efetiva e prática, da pesquisa.

A autora bell hooks ${ }^{13}$ nos ensina que a desvalorização da mulher negra ocorreu (e ainda ocorre) como resultado da exploração durante o período de escravidão, o que não foi alterado no decorrer dos anos, e isso teve sua influência nas mais diversas formas de sobrevivência e resistência de mulheres negras.

Patricia Hill Collins demonstra que o feminismo negro é um ativismo pautado nas histórias das mulheres contra o colonialismo e a escravidão. A autora afirma que as opressões sofridas por mulheres afro-americanas são de três dimensões interdependentes: exploração da mão de obra negra como essencial ao capitalismo; a dimensão política de opressão e negação de direitos e privilégios; e a construção de imagens, estereótipos e ideologias associadas à mulheres negras.

Além disso, não é possível falar sobre mulheres negras sem considerar os aspectos relativos à interseccionalidade, e esse é um aspecto que surge no feminismo negro canadense de Wane com foco em duas premissas fundamentais: resistência/luta e formação de uma identidade que não disperse o grupo social, mas o unifique. Para Wane, não é possível universalizar o modo de ser negro, mas é necessário se comprometer em realizar vínculos culturais e intelectuais pautados na ancestralidade africana (WANE et 
al, 2002, p. 22). Collins também faz uma abordagem semelhante pelo ponto de vista da interseccionalidade ao afirmar que há uma matriz de dominação que é caracterizada por opressões comuns que as mulheres negras sofrem oriundas do racismo, do sexismo e do classismo (COLLINS, 1999, p. 23).

Katerina Deliosky (WANE, 2013) fala de um senso de alienação provocado pelo feminismo liberal e de que há uma necessidade enorme de revolucionar as teorias tradicionais, tanto que parte do diálogo e também da crítica que a autora propõe consiste em entender o feminismo branco como algo que deve ser radicalmente transformado de modo que seja possível compreender o que nos oprime e o que nos privilegia, dentro dos marcos teóricos do feminismo.

A autora vai além ao indicar que omitir raça do debate é limitador ao próprio avanço do feminismo e evitar essa discussão é precisamente uma indicação do privilégio da brancura que existe dentro do movimento feminista ocidental. Ressalta a necessidade de introduzir o debate da interseccionalidade no feminismo, pois dar atenção à construção racial no corpo de mulheres brancas, esboçando o seu privilégio, é um passo crítico na direção de compreender como a ordem racial é normalizada e racionalizada e mais, como se conecta com a hegemonia do feminismo branco nas sociedades ocidentais.

A abordagem da autora vai no sentido de que, além de abarcar a interseccionalidade como central nas discussões do feminismo como um todo, é fundamental entender que a construção social em torno da ideia de raça afeta as mulheres negras e também afeta as mulheres brancas, pois essas não percebem que a sua branquitude é um privilégio em uma sociedade racializada como o Canadá. Ao não tematizar o poder e o privilégio de não ser negro, o feminismo tradicional reproduz condições epistemológicas e ideológicas que não permitem a percepção da complexidade associadas ao lugar social destinado às mulheres não brancas.

A prática do descolonialismo também é colocada como um meio emancipatório e de resistência, por exemplo, por Deepika Bahri (2013, p. 674) que critica o feminismo do Primeiro Mundo, principalmente quando questiona no seu artigo "O que acontece quando o pressuposto das mulheres como grupo oprimido se situa no contexto da escrita feminista ocidental sobre as mulheres do terceiro mundo?" A resposta é de que as 
próprias feministas ocidentais se tornam os "sujeitos dessa contra história", como se as mulheres de terceiro mundo jamais superassem a posição de objeto. Essa autora também critica o feminismo de primeiro mundo que tenta se apropriar do discurso das mulheres de terceiro mundo. A reflexão que faz é que por mais que mulheres negras do norte do planeta (EUA, Canadá, Europa Ocidental, por exemplo) sejam oprimidas em função de raça, gênero e classe, ainda são de Primeiro Mundo e talvez enfrentem certos desafios diferentes de mulheres oprimidas pelos mesmos aspectos e que estejam localizadas em países considerados em desenvolvimento ou de terceiro mundo. Essas observações já se faziam presentes nas obras da brasileira Lélia Gonzalez (2011).

Em suma, a leitura destes textos nos mostrou que o importante para a definição deste sujeito "mulher negra" que irá permear nossas investigações, em perspectiva comparada, é a criação de um panorama teórico que não fragmente as experiências de mulheres negras, independente de todas as demais identidades e identificações que estejam associadas as experiências descritas pelas diferentes autoras. Mas, que, ao mesmo tempo, considere as múltiplas dimensões das opressões e resistências que se associam a suas especificidades associadas aos diferentes aspectos descritos pelas diferentes autoras, cujos trabalhos apresentamos, aqui, resumidamente.

O que se observa é que ser mulher negra vai além da cor da pele (muito embora também se vincule a esse importante marcador social, sobretudo no Brasil). A cor da pele talvez seja o mais visível dos aspectos já que mesmo na definição de quem são as minorias visíveis, se pauta a diferenciação entre os caucasianos e brancos pela cor daqueles que não o são. Mas, além desse, são vários os marcadores que se interseccionam na construção deste grupo social que é alvo de diferentes modos de opressão que se cruzam e se sobrepõem. Talvez, por isso, seja totalmente inadequado e improdutivo, para fins de análise, reduzi-lo a um marcador construído por meio de uma única característica.

Ainda restou por estudar os efeitos do multiculturalismo na produção dos sentidos para as mulheres negras, no Canadá. Essa discussão será realizada em 2019, mas sabemos que o processo de escravização e de inclusão/exclusão dos ex escravizados, as políticas de segregação, o lugar destinado a políticas imigratórias e, sobretudo, as ideologias 
nacionais construídas em ambos os países são elementos importantes na configuração do ser mulher negra com um sujeito político.

Esse foi o aprendizado que orientou e tem orientado nossas investigações no Canadá e que permitirá extrapolar os limites do projeto inicialmente apresentado.

Assuminos que, em todos os momentos, iríamos considerar a multiplicidade de definições para mulher negra, sem contudo, permitir que essa multiciplicidade desarticulasse o eixo principal que se fez presente em todas as autoras que tematizam sobre o assunto: a ideia da diversidade somadas ao eixo que permite identificar e localizar as opressões associadas a cor, raça, etnia, local de origem, família, status, cultura, como também, a construção do sentido de mulher negra como aquela que luta contra essas opressões, luta pela liberdade e pela emancipação.

\section{1 - Formulação de subprojetos de pesquisa}

Outro desafio que se apresentou foi o de conciliar os métodos e técnicas propostos com o dia a dia de trabalho feito pelas estudantes/pesquisadoras.

Como apresentamos, o projeto aprovado tinha como foco investigar se mulheres negras construíram ou desejariam construir estratégias de enfrentamento de experiências de discriminação por raça e gênero e quais seriam essas estratégias. No Canadá, se deveria realizar pesquisa no CIARS para a compreensão do que já teria sido produzido sobre o tema.

Contudo, após a consideração dos aspectos teóricos aqui mencionados, houve a necessidade de adequar os objetivos, os métodos e as técnicas para que pudéssemos encontrar informações sobre as discriminações vivenciadas por diferentes mulheres do grupo de minorias visíveis e, dentre elas, pudéssemos selecionar as que mais se assemelhariam com as experiências vivenciadas pelas mulheres negras brasileiras.

Deparamo-nos, também, com a impossibilidade de encontrar material sistematizado sobre discriminações raciais, no Canadá, já que não há legislações voltadas a isso com o mesmo teor da lei que existe no Brasil.

Cogitamos que uma solução possível para sabermos sobre as experiências vividas pelas mulheres, no Canadá, seria conversar diretamente com elas e ouvir delas o que 
pensavam. Contudo, como a proposta inicial do projeto não contemplava a realização de entrevistas fora do Brasil, descobrimos que não poderíamos realizá-las, já que a universidade parceria (assim como a USP) é bastante rigorosa no que diz respeito a seus protocolos éticos para a condução de pesquisas e não havia autorização, no projeto encaminhado, para o uso desta técnica de pesquisa. Somente poderíamos realizar pesquisa documental e bibliográfica.

Diante desses obstáculos, decidimos por reorganizar o projeto principal em diferentes subprojetos que permitissem que cada estudante aprofundasse uma investigação específica acerca de aspectos que, somados, poderiam nos oferecer o panorama da situação vivenciada pelas mulheres negras, no Canadá. Assim passamos a considerar a proposta do projeto principal como um projeto guarda-chuva, com diferentes subprojetos a serem desenvolvidos ao longo dos meses.

Para chegar às informações que precisávamos obedecendo os limites do projeto proposto e aprovado, especificamos um pouco mais o que poderia ser obtido por meio de pesquisa documental que mostrasse como as discriminações/opressões seriam tratadas (ou não tratadas) pelo Estado por meio de políticas, políticas públicas e legislações, Como seriam tratadas (ou não tratadas) por órgãos e agências? Como surgiriam em textos sobre o tema?

Deste modo, todas as estudantes se dedicariam à pesquisa bibliográfica sobre estudos pós-coloniais e feminismo pós-colonial. Mas, além disso, uma delas, Camila Tavares de Moura Brasil Matos, se dedicou à pesquisa em documentos da área do Direito/direitos humanos buscando compreender a construção dos direitos das mulheres das minorias visíveis (já que aprendemos sobre as dificuldades de encontrar documentos oficiais que se voltassem, especificamente, às mulheres negras no mesmo sentido dado ao termo, no Brasil).

Essa pesquisadora buscou compreender por meio de quais instrumentos os direitos das mulheres negras, no Canadá, seriam protegidos. Para isso utilizou parâmetros de diferentes níveis de proteção de Direitos Humanos no país, para poder pensar como seriam analisados os casos de discriminação contra esse grupo de mulheres (considerando o conceito de minorias visíveis). Buscou informações sobre como as mulheres (ou seus 
representantes legais) exporiam os casos de discriminação. Deste modo, teríamos acesso às experiências relatadas como discriminatórias, desrespeitosas ou opressivas.

Outra pesquisadora, Bárbara Santos de Araújo, se concentrou em questões pedagógicas buscando propostas na área das políticas públicas da educação que se voltasse para o tema da diversidade. Neste caso, a estudante deu início a uma pesquisa exploratória sobre as propostas de educação formuladas pelo Toronto District School Board (TDSB), órgão responsável pela gestão de políticas educacionais, para que pudesse verificar de que modo os temas do respeito às mulheres negras seriam tratados. Ao final deste percurso seria possível observar quais seriam as demandas, estratégias e experiências apresentadas por esse grupo no que tange aos conteúdos que defendem devam ser ministrados nas escolas.

A terceira pesquisadora, Eloya Porto da Rocha, se dedicou a investigar os atos do legislativa canadense destinados a políticas antidiscriminatórias. Analisou inúmeros documentos para conhecer a participação de mulheres negras canadenses neste espaço propositivo para mapear a sua representatividade na construção de estratégias de defesa dos direitos deste grupo social.

Por fim, a partir da análise dos casos de injúria racial que compuseram o banco de dados do projeto foi feita uma discussão, por Gislene Aparecida dos Santos, sobre as diferentes dimensões de opressão que afetam as mulheres negras brasileiras, com sugestões acerca do que se deve considerar quando se pesquisa no tema, nas ciências sociais e jurídicas.

Cada projeto observou modos de enfrentamento da discriminação de raça e gênero e contribuiu para responder à pergunta orientadora do projeto principal proposto. No momento, cada pesquisadora, individualmente ou em parceria, se dedica a finalizar a pesquisa iniciada e a produzir material escrito por meio do qual seus resultados se tornem conhecidos.

Esses foram os projetos desenvolvidos no primeiro ano de pesquisa. Com esse aprendizado, elaboramos subprojeto para o segundo ano de trabalho que ampliará, ainda mais, a compreensão do tema aqui apresentado. 


\section{Conclusão}

Após a realização do primeiro ano de investigação, pudemos compreender que, mesmo quando se trata de um tema circunscrito à situação de opressão e as estratégias contra ela construídas por mulheres negras, considerando essa especificidade, há que se observar os impactos das culturas e políticas locais na definição e criação de categorias. Não se pode falar sobre mulheres negras de modo universal, a-histórico e não considerando as realidades de cada local para a definição das categorias para a análise do que é observado.

Então, o pressuposto do qual partimos segundo o qual haveria uma comunidade de destino que assemelharia as experiências vivenciadas por todas as mulheres negras, independente de sua localização geográfica, somente pode ser assumido de modo muito parcial já que o local de origem, de vida, a cultura, as dinâmicas políticas e culturais são ingredientes essenciais na definição do modo como cada um constrói sua identidade e age diante das opressões que surgem na sociedade.

Além deste aspecto, ao longo da investigação, percebemos que o projeto apresentado desconsiderava importantes fatores associados a questões de poder e das lutas por emancipação. As estratégias de enfrentamento não podem se concentrar, exclusivamente, em questões de saúde física e mental, que são importantes, mas não são suficientes para alterarem a realidade social. Até mesmo o modo de se conceiturar o que seja saúde mereceria reflexão. A depender do enfoque dado para o termo saúde e bem estar isso pode significar considerar apenas aspectos individuais e enfatizar que cada mulher seria, per si, a responsável por encontrar modos de superar a opressão da qual é vítima. Então, teríamos que discutir os sentidos para saúde e bem estar das mulheres negras, mas sobretudo investigar os aspectos associados a produção das opressões que foram estruturadas, como afirmam os estudos pós-coloniais, desde a Modernidade.

Sendo assim, e com base nos estudos do feminisno negro e nos estudos pós-coloniais que orientam nossas investigações, sabemos que as estratégias para enfrentamento das discriminações multidimensionadas devem ser construídas de modo a envolver a todas as pessoas que têm como objetivo a transformação da sociedade. Não se pode lançar somente sobre as mulheres negras (ou as minorias visíveis) o fardo para essa transformação. Contudo, é observando as múltiplas opressões que recaem sobre esse 
grupo que conseguimos compreender, de modo aprofundado, as dinâmicas da produção das desigualdades, das violências e das explorações ao longo dos séculos e nas várias localidades no globo já que são as mulheres negras as que se encontram nos estratos com os maiores índices de vulnerabilidade social.

Essas considerações são fundamentais para que se perceba a importância da participação de pessoas de outros grupos sociais na construção de estratégias de enfrentamento das opressões aqui descritas. Essas participações devem ser temperadas pela compreensão do lugar que ocupam nas diferentes sociedades.

Por fim, percebemos que há modos de subalternizações que intersecionam as localidades e as opressões que ocorrem dentro dos mesmos grupos sociais. Pessoas de países considerados em desenvolvimento não recebem o mesmo tratamento que as pessoas dos países desenvolvidos. Acadêmicos de Terceiro Mundo em universidades de Primeiro Mundo não desfrutam do mesmo status e reconhecimento que acadêmicos do Primeiro Mundo, mesmo que, em princípio e em uma leitura menos profunda, se possa dizer que pertencem ao mesmo grupo social de mulheres negras. Há diferenças a serem observadas entre as mulheres negras e das minorias visíveis em acordo com o status que ocupam nas universidades que são, também, espaços de poder.

Por isso, tivemos que considerar que há algumas estratégias de enfrentamento das opressões que podem ser assumidas em todos os países das Américas (incluímos entre elas, aquelas que aparecem descritas nos textos das maiorias das autoras do feminismo negro aqui apresentadas e que dizem respeito aos desafios da transformação social considerando os sistemas interligados de poder como o sexismo, o classismo, o racismo e o patriarcado)

Contudo, há estratégias que apontam e devem apontar para a especificidade da vida das mulheres negras nos países empobrecidos pelos sistemas de exploração e opressão do colonialismo e dos imperialismos praticados pelos países do norte ocidental sobre os países do sul. Ou seja, estratégias que dizem respeito, mais diretamente, aos países da América Latina.

Pensando especificamente nos espaços de construção do conhecimento, cabe considerar a diferença de agenda das mulheres que atuam no Canadá e EUA (mesmo que 
provenientes de outros locais) da agenda de todas as outras mulheres das Américas. O Ocidente e o resto, diria um autor (The west and the rest).

Na perspectiva do feminismo negro e dos estudos pós-coloniais, as mulheres negras da América Latina estariam lutando pela descolonização dos saberes que são endossados e divulgados nas universidades de Primeiro Mundo. Estariam buscando e construindo novas epistemologias e novos sentidos para o conhecimento que deslocariam os saberes dos centros, para as margens e desestabilizariam os poderes construídos nas universidades do norte do mundo. Por outro lado, as mulheres negras atuantes nas universidades de Primeiro Mundo, obviamente, não desejariam se ver desapropriados dos lugares de poder que duramente conquistaram nestes espaços. Sendo assim, como construir estratégias de descolonização além das fronteiras? Como investir na solidariedade quando parte dela significa abrir mão de conquistas que não foram fáceis de se obter? Essas foram algumas das questões que ainda não pudemos responder.

Todas essas diferenças e dimensões impactam na produção e concretização de parcerias internacionais e precisam ser consideradas no momento da realização de propostas para que os projetos se desenvolvam de modo adequado. Todas elas se apresentaram a nós como desafios a serem transpostos e, hoje, mais do que ontem, sabemos que há um enorme caminho a ser percorrido quando o tema é a equidade.

\section{Referências}

ALVAREZ, S. e. Para além da so ciedade civil: reflexões sobre o campo feminista. Cadernos pagu, (43), 2014, 13-56. Disponível em: https://dx.doi.org/10.1590/01048333201400430013.

BAHRI, D. Feminismo e/no pós-colonialismo. Revista Estudos Feministas, 21(2), 2013, 659-688. Recuperado de https://periodicos.ufsc.br/index.php/ref/article/view/ s0104026x2013000200018. 2013.

BRAND, D. No Burden to Carry: Narratives of Black Working Women in Ontario 1920s to 1950 s (excerpts). In: No Burden to Carry Narratives of Black Working Women in Ontario 1920s to 1950s, pp.20-28. Toronto: Women's Press, 1991.

CARTY, L; BRAND, D. Visible Minority Women: A Creation of the Canadian State. In: The Gaze: Returning the Gaze, Essays on Racism, Feminism and Politics. Toronto: Sister Vision Press: Black Women and Women of Colour Press, 1993, p. 207-222. 
CARTY, L; BRAND, D. Black Women in Academia: A Statement from the Periphery. In: Unsettling Relations: The University as a Site of Feminist Struggle. Himani Bannerji et. al.(Ed), 13-41. Toronto: Women's Press, 1991.

CRAWFORD, C. Public policy and private decisions: The evolutionary context. In: C. Crawford \& C. Salmon (Ed), Evolutionary psychology, Public policy and Private Decisions. Mahwah, NJ: Lawrence Erlbaum, 2004.

COLLINS, P. H. Black Feminist Thought: Knowledge, Consciousness, and the Politics of Empowerment. New York: Routledge Classics, 1999.

DAVIS, A. Mulheres, raça e classe. São Paulo: Boitempo, 2016.

DEI, G.; CALliSTE, A. Power, Knowledge and Anti-Racism Education. A Critical Reader. Halifax: Fernwood Publishing, 2000.

DELIOVSKY, K. Transgressive Whiteness: The Social Construction of White women Involved in Interracial Relationships with Black Men. In: Njoki Wane, Katerina Deliovksy, \& Erica Lawson, (Ed) Back to the Drawing Board: African Canadian Feminisms. Toronto: Sumach Press, 2002.

ELABOR-IDEMUDIA, P. Equity Issues in the Academy: An Afro-Canadian Women's Perspective. In: Journal of Negro Education. Black Women in the Academy: Challenges and Opportunities. Vol. 70 (3), 2001, p. 192-203.

FANON, F. Pele Negra. Máscaras Brancas. Rio de Janeiro: Fator, 1983.

FONSECA, F. de C. A. et al. A influência de fatores emocionais sobre a hipertensão arterial. In: Jornal Brasileiro de Psiquiatria Rio de Janeiro, v. 58, n. 2, 2009.

GONZALEZ, L. Por um feminismo afrolatino-americano. Disponível em: https://edisciplinas.usp.br/pluginfile.php/271077/mod_resource/content/1/Por\%20um\%20feminismo\%20Afro-latino-americano.pdf

GREER, T. M. Coping strategies as moderators of the relation between individual racerelated stress and mental health symptoms for African American Women. In: Psychology of Women Quartely, 35 (2), 215-226, 2011.

HANCOCK, A. Intersectionality: An Intellectual History. New York: Oxford University Press, 2016.

HENRY, B. Report of the CIC Chair 1997-1998, Canandian Chichal. News, 50 (7): 23, 1998.

HOOKS, B. Ain't I a Woman: Black Women and Feminism. New York: Routledge Classics, 1981.

JAMES, C.E.; SHADD, A. Talking About Difference: Encounters in Culture, Language and Identity. Toronto: Between The Lines, 1994.

KESLER, C. ; SCHWARTZMANN, L. F. From Multi-Racial Subjects to Multi-Cultural Citizens: Social Stratification and Ethnoracial Classification among Children of Immi- 
grants in the United Kingdom. In: International Migration Review, Volume 49, Issue 3, pages 790-836, 2015.

LAWSON, E. Images in Black: Black women, Media and the Mythology of an Orderly Society. In: Njoki Wane, Katerina Deliovsky, \& Erica Lawson. (Ed). Back to the Drawing Board: African Canadian Feminisms. Toronto: Sumach Press, 2002.

MACHADO, M. C.; PIRES, C. G.; LOBÃO, W. M. Concepções dos hipertensos sobre os fatores de risco para a doença. Ciência Saúde Coletiva. Rio de Janeiro, v. 17, n. 5, maio 2012 .

MAIA, S. Sedução e identidade nacional: dançarinas eróticas brasileiras no Queens, Nova York. Revista Estudos Feministas, 17(3), 769, 2009. Disponível em: dehttps://periodicos. ufsc.br/index.php/ref/article/view/S0104-026X2009000300009

MAKEDA, S. The Issue is 'ism: Women of Colour Speak Ou. Fireweed's issue 16. Toronto, On: Sister Vision, 1989.

MASSAQUOI, N. An African Child Becomes a Black Canadian Feminist: Oscillating Identities in the Black Diaspora. In: Canadian Woman Studies. Vol 23.N. 2p.140-144, 2004.

MASSAQUOI N.; WANE, N. Theorizing empowerment: Canadian perspectives on feminist thought. Toronto: Innana Publishers, 2007.

MATOS, Camila Tavares de Moura Brasil. A percepção da injúria racial e racismo entre os operadores do Direito. 200 f. Dissertação (Mestrado em Direitos Humanos) - Programa de Pós Graduação em Direitos Humanos, Universidade de São Paulo, São Paulo, 2016.

NASCIMENTO, J. S.; SARDINHA, A. H. de L. de; PEREIRA, A. N. S. Risco cardiovascular em mulheres negras portadoras de hipertensão arterial em uma comunidade de São Luís- MA. Saúde Coletiva, vol. 9, núm. 56, 2012, pp. 40-45.

OPINI, B.; WANE, N. When Race Structures "Being-Ness". The Experiences of Black women in Canada. In: Massaquoi \& Njoki Wane (ed), Theorizing Empowerment: Black Canadian Feminist Thought. Toronto, On.: Innana Publishers, 2007.

RIVIERE, D. Adventures of a Black Girl in Search of Herself: Some Thoughts on Canadian Feminisms. In: Hecate 30 (1) pp. 222-230, 2004.

SAMPAIO, A. S. Ecos da hipertensão: a vivência de mulheres negras no Rio de Janeiro. Mestrado. Pontifícia Universidade do Rio de Janeiro. Programa de Pós-graduação em Psicologia Clínica. 2009.

SANTOS, G. A. dos. Selvagens, exóticos, demoníacos: idéias e imagens sobre uma gente de cor preta. Estudos Afro-asiáticos, Rio de Janeiro, 24(2), 275-289, 2002a.

SANTOS, G. A. A invenção do ser negro. São Paulo/Rio de Janeiro: Pallas/FAPESP/ EDUC, 2002b.

SANTOS, G. A. Mulher negra. Homem branco. Rio de Janeiro: Pallas, 2004.

SANTOS, G. A. Percepções da diferença, São Paulo, Brasil: MEC, 2007. 
SANTOS, G. A. Racism and Its Masks in Brazil: On Racism and the Idea of Harmony, In: Branche, Jerome (ed.). Race, Colonialism, and Social Transformation in Latin America and Caribbean, Gainesville: University Press of Florida, 91 -115, 2008.

SANTOS, G. A. Reconhecimento, utopia, distopia. São Paulo, Brasil, Annablume/ FAPESP, 2012.

SANTOS, G. A. dos. Nem crime, nem castigo: o racismo na percepção do judiciário e das vítimas de atos de discriminação. Revista Do Instituto de Estudos Brasileiros, 2015 https://doi.org/10.11606/issn.2316-901X.v0i62p184-207

SANTOS, G. A. dos, NOGUTI, H. H., \& MATOS, C. T. M. B. Racismo ou não? A percepção de estudiosos do direito sobre casos com conteúdos racistas. Revista de Estudos Empíricos Em Direito, 1(2), 2014. https://doi.org/10.19092/reed.v1i2.35

SMEDLEY, A. Race in North America: Origin and Evolution of a Worldview. New York: Routledge, 1993.

TASTSOGLOU, E. Race and the Politics of Personal Relationships: Focus on Black Canadian Women. AFFLIA 17 (1), 93-111, 2002.

WANE, N. N., DELIOVSKY, K.; LAWSON, E. Back to the drawing board: African Canadian feminisms. Toronto: Sumach Press, 2002.

. Carving out Critical Space: Black Women and the Academy" in: Wane, Deliovsky, K \& Lawson, E (Rd) Back to the Drawing Board: African Canadian Feminisms. Toronto: Sumach Press, 2002, p. 175-198.

. Black Canadian Feminist Thought: Drawing from Experiences of my Sisters. In: Wane, N. Deliovsky, K \& Lawson, E (Ed) Introduction. Back to the Drawing Board: African Canadian Feminisms. Toronto: Sumach Press, 2002.

. Black Canadian Feminist Thought: Tensions and Possibilities. Canadian Woman Studies. Vol 23. No.2. p.145, 2004.

African Women and Canadian History: A Black Feminist Articulation. In: Notisha Massaquoi \& Njoki Wane (Ed), Theorizing Empowerment: Black Canadian Feminist Thought. Toronto: Innana Publishers, 2007.

. Black Canadian Feminist Thought: Imagining New Possibilities, In: Massaquoi \& Njoki Wane (Ed), Theorizing Empowerment: Black Canadian Feminist Thought. Toronto: Innana Publishers, 2007.

. NEEGAN, E. “African Women's Indigenous Spiritual Knowledge. In: Massaquoi \& Njoki Wane (Ed), Theorizing Empowerment: Black Canadian Feminist Thought. Toronto: On, Innana Publishers, 2007.

. Interview with Zulu. Practices of African Women's Spirituality. In: Massaquoi \& Njoki Wane (Ed), Theorizing Empowerment: Black Canadian Feminist Thought. Toronto: Innana Publishers, 2007. 
. Black Canadian Feminist Thought: Perspectives on Equity \& Diversity in the Academy Journal of Race, Ethnicity and Education, 2008a.

. Uncovering the Well: Black Feminism in Canada. New Dawn: Journal of Black Canadian Studies, 2008b.

et al. A glance at Africa. Indiana: Authorhouse, 2009.

. (under review) African Canadian Women and the Criminal Justice System: An Overview. Race, Gender \& Class Journal. Southern University at New Orleans. US.

. Ruptures: Anti-Colonial \& Anti-Racist Feminist Theorizing. Toronto: Sense Publishers, 2013.

WEST, L, DONOVAN, R., ROEMER, L. Coping with racism: what works and doesn't work for Black women? Journal of Black Psychology, 36, 2010, p . 331-349.

ZAMBRANO, C. G. Mulheres negras em movimento: ativismo transnacional na América Latina (1980-1995). Tese de Doutorado, Faculdade de Filosofia, Letras e Ciências Humanas, Universidade de São Paulo, São Paulo, 2017. Doi:10.11606/T.8.2018.tde-20022018-

\section{Notas}

1 Professora da Escola de Artes, Ciências e Humanidades (EACH-USP), docente do Programa de pós-graduação em Direitos Humanos da Faculdade de Direito (FD-USP), pesquisadora do Diversitas - Núcleo de Estudos das Diversidades, Intolerâncias e Conflitos (USP). Orienta e coordena o projeto descrito no artigo, foi responsável pela apresentação do projeto neste texto e na composição do artigo. Email: gislene@usp.br

2 Camila Tavares de Moura Brasil Matos é doutoranda em Direitos Humanos pela Faculdade de Direito da Universidade de São Paulo. Participou da primeira etapa da pesquisa na University of Toronto e auxiliou com a redação deste artigo. Email: camila.tmbm@gmail.com

3 FAPESP - Fundação de Amparo à Pesquisa do Estado de São Paulo. A pesquisa mencionada foi financiada pela FAPESP - Fundação de Amparo à Pesquisa do Estado de São Paulo, por meio do processo 2010/12609-1. A pesquisa em desenvolvimento está sendo realizada com apoio da Coordenação de Aperfeiçoamento de Pessoal de Nível Superior - Brasil (CAPES) - Código de Financimento 001

4 Utilizamos a palavra vítima porque é este o termo utilizado nos documentos que constam de nossa base de dados. Não temos intenção de que o uso desta expressão indique a vitimização desses sujeitos.

5 GEPPIS é o Grupo de Estudos e Pesquisas das Políticas Públicas para a Inclusão Social, da Escola de Artes, Ciências e Humanidades (EACH-USP), coordenado pela proponente do projeto. Website: http://each.uspnet. usp.br/web/prof/geppis

${ }^{6}$ Há algumas publicações nas quais apresentamos a metodologia assumida para a composição do banco de dados formado e sobre os achados desta pesquisa. Para isso, seria interessante consultar: Santos (2015), Santos, Matos e Noguti (2014) e Matos (2016).

7 Para fins deste artigo, "coping" significa enfrentamento ou forma de lidar com dificuldades.

${ }^{8}$ Tradução do original: "Estratégias de enfrentamento refere-se aos esforços específicos, tanto comportamentais como psicológicos, que as pessoas usam para minimizar eventos estressantes".

9 Tradução do original: "(...) members of visible minorities means persons, other than aboriginal peoples, who are non-Caucasian in race or non-white in colour”. Retirado de http://laws-lois.justice.gc.ca/eng/acts/E-5.401/ section-3.html. Minorias visíveis ou invisible minorities é um termo oficial usado pelo Governo do Canadá para designar grupos minoritários de sua população. É utilizado nas legislações e na criação e implementação de políticas públicas.

${ }^{10}$ As informações sobre o censo e sobre minorias visíveis podem ser todas encontradas na página do Governo Canadense: <http://www.statcan.gc.ca/eng/start>

${ }^{11}$ Tradução do original: “(...) theoretical tool meant to elucidate and analyze the historical, social, cultural and economic relationships of woman of african descent as the basis for development of a liberatory práxis. 
${ }^{12}$ Sabemos que, no Brasil, a questão de identificação de pessoas que se autodeclaram negras também é um terreno em constante disputa. Não entraremos nesta discussão neste artigo, mas o tema das políticas afirmativas por meio da autodeclaração de quem seria preto ou pardo gerou enorme debate no cenário político e acadêmico por anos a fio.

${ }^{13}$ bell hooks é o pseudônimo da autora estadunidente Gloria Jean Atkins. O seu pseudônimo é grafado em letras minúsculas, pois a autora entende que a atenção deve ser dada ao conteúdo da sua escrita e da sua carreira e não ao seu nome. 